\title{
Physical constraints on the coefficients of Fourier expansions in cylindrical coordinates
}

\author{
H. Ralph Lewis ${ }^{\text {a) }}$ \\ Los Alamos National Laboratory, Los Alamos, New Mexico 87545 \\ Paul M. Bellan \\ California Institute of Technology, Pasadena, California 91125
}

(Received 12 March 1990; accepted for publication 20 June 1990)

\begin{abstract}
It is demonstrated that (i) the postulate of infinite differentiability in Cartesian coordinates and (ii) the physical assumption of regularity on the axis of a cylindrical coordinate system provide significant simplifying constraints on the coefficients of Fourier expansions in cylindrical coordinates. These constraints are independent of any governing equations. The simplification can provide considerable practical benefit for the analysis (especially numerical) of actual physical problems. Of equal importance, these constraints demonstrate that if $\mathbf{A}$ is any arbitrary physical vector, then the only finite Fourier terms of $A_{r}$ and $A_{\theta}$ are those with $m=1$ symmetry. In the Appendix, it is further shown that postulate (i) may be inferred from a more primitive assumption, namely, the arbitrariness of the location of the cylindrical axis of the coordinate system.
\end{abstract}

\section{INTRODUCTION}

In mathematical physics, changing from one set of independent variables to a different, but equivalent, set is a matter of convenience only, since all physical results must be independent of the choice of coordinate system. Frequently, the choice of independent variables is motivated by some symmetry of the problem. For example, if there is a line source, or if boundary conditions are specified on a circular cylinder, then cylindrical coordinates are likely most appropriate. However, the choice of a particular set of independent variables (e.g., the $r, \theta, z$ of cylindrical coordinates) might inadvertently introduce mathematically allowable but physically unrealistic terms-e.g., singularities at the axis. These nonphysical terms must be eliminated by the imposition of physical constraints on the mathematical solutions.

Let us briefly review two traditional methods by which these constraints are imposed.

(1) Analytic method: First, a set of equations relevant to the problem at hand is derived; second, general mathematical solutions to these equations are found; third, some subset of these solutions is discarded as being nonphysical, and finally, boundary conditions are used to determine a suitable combination of the remaining physically allowable solutions to describe the specific problem.

(2) Numerical method: Again, a set of equations relevant to the problem is derived; second, the space in which the problem is to be solved is quantized into grid points; third, the equations are put into discrete form so as to establish a numerical algorithm; fourth, a numerical solution is developed using the algorithm. Mathematically allowable but nonphysical solutions are eliminated by constraints determined in an ad hoc manner. For example, a simplified but local analytic solution might be developed in the neighbor-

\footnotetext{
a) Temporary address: U.S. Department of Energy, Office of Energy Research, Office of Fusion Energy, Washington, DC 20545.
}

hood of the axis, and then grafted onto the numerical solution outside this region.

.A well-known example of the analytical method is where one Fourier analyzes in $z$ and $\theta$ linear partial differential equations so as to obtain an ordinary differential equation in $r$ for the Fourier coefficients. Physical constraints are imposed in order that solutions of the equations be regular in the region of interest. The most obvious example of this method is that involving Bessel's equation: Although both the $J_{m}$ and $Y_{m}$ Bessel functions satisfy Bessel's equation, for physical problems including the axis, one rejects the $Y_{m}$ solution because the $Y_{m}$ solution is singular there.

The point we wish to make in this paper is that the physical constraints are more fundamental than the differential equations and can be determined without reference to differential or any other equations. In this paper we restrict our attention to cylindrical coordinates. However, similar considerations apply to all variable transformations, those of a nongeometrical nature as well as those of a geometrical nature.

There are three reasons why it is important for one to be able to determine the constraints without reference to any governing equations. First, even if governing equations are known and could, in principle, be used to derive the constraints (e.g., by rejecting singular solutions), it is generally more convenient to be able to assume from the outset the form required of each quantity. In numerical computation of physical problems especially, singular nonphysical terms are a serious difficulty since small finite differencing or truncation errors easily excite the unwanted terms. Because these nonphysical terms are divergent, they mask the desired physical solution and render the computation useless. It is clearly essential in these situations to be able to invoke constraints that eliminate the unwanted divergences. (Often in contemporary work, if a problem is sufficiently complicated, numerical analysts resort to the messy stratagem of solving 
the problem using a less appropriate coordinate system solely to avoid difficulties with these nonphysical singularitiese.g., Cartesian coordinates are often used for problems that have cylindrical symmetry simply to avoid singularities at the axis. Clearly, it would be preferable to be able to invoke appropriate physical constraints and so use the more natural cylindrical coordinates.)

Second, although the governing equations might be known, they may be so complicated that it is not feasible to use them to provide constraints. Indeed, the considerations in this paper originated from a numerical computation problem in plasma physics where the governing equations were integrodifferential equations in a cylindrical coordinate system. Because these equations were nonlocal, it was not possible to determine the behavior of solutions at the axis and so guarantee regularity.

Third, it may be that the governing equations are unknown or even that a solution to the governing equations is not sought. The results of this paper have been applied ${ }^{1}$ to reduce in a most substantial and significant manner the complexity of the theoretical description of a magnetohydrodynamic dynamo problem where the precise form of the governing equations was not well established.

\section{SYMMETRY CONSTRAINT}

Before we launch into our discussion of regularity, it is worthwhile to derive a purely mathematical symmetry constraint on Fourier coefficients. Later, we will combine this result with the regularity analysis to determine the least restrictive physically permissible Fourier coefficients.

We consider the transformation between Cartesian coordinates $(x, y, z)$ and cylindrical coordinates $(r, \theta, z)$ given by

$$
x=r \cos \theta, \quad y=r \sin \theta .
$$

In our considerations here and in the rest of this paper the $z$ coordinate will never play a role; thus we hold it constant and henceforth suppress it in the notation. The Cartesian coordinates of a point are not changed by replacing $r$ by $-r$ and $\theta$ by $\theta+\pi$ in Eq. (1). This symmetry of the transformation requires that any point function of $(r, \theta)$, say $f(r, \theta)$, satisfy

$$
f(r, \theta)=f(-r, \theta+\pi) .
$$

If we make a Fourier series representation of $f(r, \theta)$,

$$
f(r, \theta)=\sum_{m=-\infty}^{\infty} a_{m}(r) e^{i m \theta},
$$

we see that in order to satisfy Eq. (2), we must have

$$
a_{m}(r)=(-1)^{m} a_{m}(-r) .
$$

In words, if $m$ is even, then $a_{m}$ is an even function of $r$, whereas if $m$ is odd, then $a_{m}$ is an odd function of $r$. This symmetry result also restricts the form of physically allowed linear differential equations for $a_{m}(r)$ to be such as not to not alter the parity of $a_{m}(r)$; all terms in a linear differential equation must be odd in $r$ or else all even in $r$ (if the equation is nonlinear, then this restriction need not apply).

\section{REGULARITY CONSTRAINT: SCALARS}

Let us assume that $\Psi(r, \theta)$ is a physical scalar, regular at $r=0$, and express $\Psi$ as a Fourier series with respect to $\theta$. Thus we write

$$
\Psi(r, \theta)=\sum_{m=-\infty}^{\infty} a_{m}(r) \exp (\operatorname{im} \theta)=\sum_{m=-\infty}^{\infty} \Psi_{m}(r, \theta) .
$$

We now show that $a_{m}(r)$ must have a very specific type of $r$ dependence. We use the assumption that each coefficient $\Psi_{m}(r, \theta)$ is a regular (i.e., infinitely differentiable) function of $(x, y)$ at $r=0$. The function $\exp (\operatorname{im} \theta)$ is not a regular function of $(x, y)$ at $r=0$. However, the function $[r \exp ( \pm i m \theta)]^{|m|}=(x \pm i y)^{|m|}$ is obviously a regular function of $(x, y)$ because it is a polynominal in $(x, y)$. We express $\Psi_{m}$ as

$$
\begin{aligned}
\Psi_{m} & =a_{m}(r) \exp (i m \theta)=\left[a_{m}(r) / r^{|m|}\right][r \exp ( \pm i \theta)]^{|m|} \\
& =\left[a_{m}(r) / r^{|m|}\right](x \pm i y)^{|m|},
\end{aligned}
$$

where the + sign is used if $m>0$ and the - sign is used if $m<0$. Since $(x \pm i y)^{|m|}$ is regular, we must require that $a_{m}(r) / r^{|m|}$ be regular. For $a_{m}(r) / r^{|m|}$ to be nonsingular, we must require

$$
a_{m}(r) \sim r^{|m|} \text { as } r \rightarrow 0 .
$$

The symmetry constraint of Eq. (4) then gives

$$
a_{m}(r)=r^{|m|} f_{m}\left(r^{2}\right),
$$

where $f_{m}\left(r^{2}\right)$ is a regular function of $r^{2}$ and so has a Taylor expansion

$$
f_{m}\left(r^{2}\right)=f_{m}^{(0)}+f_{m}^{(2)} r^{2}+f_{m}^{(4)} r^{4}+\cdots .
$$

If odd powers of $r$ were present in Eq. (9) then, because $r=\sqrt{x^{2}+y^{2}}, f_{m}\left(r^{2}\right)$ would not be a regular function of $(x, y)$.

The analysis in this section was based on the postulate that all physical quantities when expressed in Cartesian coordinates are infinitely differentiable on the axis. It is shown in the Appendix that this postulate may be inferred from a more primitive assumption, namely, the assumption that the location of the cylindrical axis of a coordinate system for describing a physical system is arbitrary.

\section{REGULARITY CONSTRAINTS: VECTORS}

Let $A$ be a vector representing a physical quantity. We will again exploit the transformation from cylindrical to Cartesian coordinates to determine the functional form of cylindrical components of $\mathbf{A}$. We do this by writing

$$
\begin{aligned}
\mathbf{A}= & A_{r} \hat{r}+A_{\theta} \hat{\theta}+A_{z} \hat{z} \\
= & A_{r}(\cos \theta, \sin \theta, 0) \\
& +A_{\theta}(-\sin \theta, \cos \theta, 0)+A_{z}(0,0,1),
\end{aligned}
$$

in which case

$$
A_{x}=A_{r} \cos \theta-A_{\theta} \sin \theta .
$$

Let us now expand $A_{r}$ and $A_{\theta}$ in terms of their Fourier terms in $\theta$ : 


$$
\begin{aligned}
& A_{r}=\sum_{m=-\infty}^{\infty} A_{r m} \exp (i m \theta), \\
& \mathbf{A}_{\theta}=\sum_{m=-\infty}^{\infty} \mathbf{A}_{\theta m} \exp (i m \theta) .
\end{aligned}
$$

Combining Eqs. (11) and (12), we obtain

$$
\begin{aligned}
A_{x}= & \frac{1}{2} \sum_{m=-\infty}^{\infty}\left[\left(A_{r m}+i A_{\theta m}\right) e^{i(m+1) \theta}\right. \\
& \left.+\left(A_{r m}-i A_{\theta m}\right) e^{i(m-1) \theta}\right]
\end{aligned}
$$

or

$$
\begin{aligned}
A_{x}= & \frac{1}{2} \sum_{m=1}^{\infty}\left[\left(A_{r m}+i A_{\theta m}\right) e^{i(|m|+1) \theta}+\left(A_{r m}-i A_{\theta m}\right) e^{i(|m|-1) \theta}\right]+\frac{1}{2}\left[\left(A_{r 0}+i A_{\theta 0}\right) e^{i \theta}+\left(A_{\circ}-i A_{\theta 0}\right) e^{-i \theta}\right] \\
& +\frac{1}{2} \sum_{m=-1}^{\infty}\left[\left(A_{r m}+i A_{\theta m}\right) e^{-i(|m|-1) \theta}+\left(A_{r m}-i A_{\theta m}\right) e^{-i(|m|+1) \theta}\right] .
\end{aligned}
$$

We may rewrite Eq. (13b) as

$$
\begin{aligned}
A_{x}= & \frac{1}{2} \sum_{m=1}^{\infty}\left[\left(A_{r m}+i A_{\theta m}\right) \frac{(x+i y)^{|m|+1}}{r^{|m|+1}}+\left(A_{r m}-i A_{\theta m}\right) \frac{(x+i y)^{|m|-1}}{r^{|m|-1}}\right] \\
& +\frac{1}{2}\left[\left(A_{r 0}+i A_{\theta 0}\right) \frac{(x+i y)}{r}+\left(A_{r 0}-i A_{\theta 0}\right) \frac{(x-i y)}{r}\right] \\
& +\frac{1}{2} \sum_{m=-1}^{\infty}\left[\left(A_{r m}+i A_{\theta m}\right) \frac{(x-i y)^{|m|-1}}{r^{|m|-1}}+\left(A_{r m}-i A_{\theta m}\right) \frac{(x-i y)^{|m|+1}}{r^{|m|+1}}\right] .
\end{aligned}
$$

We require each term in Eq. (14) to be regular. Only positive powers of $x \pm i y$ occur and these factors are all regular. Thus we must require the regularity of the remaining factors. We will consider $m>0, m=0$, and $m<0$ separately.

Case (i), $m=0$ : Here, we require that $\left(A_{r_{0}} \pm A_{\theta 0}\right) / r$ be regular, so that we must have both $A_{, 0} \sim r$ and $A_{\theta 0} \sim r$ as $r \rightarrow 0$. both

Case (ii), $m>0$ : For regularity we must have, as $r \rightarrow 0$,

$$
A_{r m}+i A_{\theta m} \sim r^{p}, \text { where } p \geqslant|m|+1
$$

and

$$
A_{r m}-i A_{\theta m} \sim r^{q}, \text { where } q \geqslant|m|-1,
$$

since both these terms occur for $m>0$. We could let $p=q=|m|+1$, but this is not the least restrictive possibility. To obtain the least restrictive possibility, we satisfy Eq. (15b) by letting $q=|m|-1$ with both $A_{r m} \sim r^{|m|-1}$ and $A_{\theta m} \sim r^{|m|-1}$ as $r \rightarrow 0$. However, Eq. (15a) will then be violated unless we set $A_{r m}+i A_{\theta m}=0$ for terms of order $r^{|m|-1}$. Thus the least restrictive allowable form is

$$
\begin{aligned}
& A_{r m}=\lambda_{m} r^{|m|-1}+r^{|m|+1} g_{m}\left(r^{2}\right), \\
& A_{\theta m}=i \lambda_{m} r^{|m|-1}+r^{|m|+1} h_{m}\left(r^{2}\right),
\end{aligned}
$$

where $\lambda_{m}$ is a constant and $g_{m}\left(r^{2}\right)$ and $h_{m}\left(r^{2}\right)$ are regular functions of $r^{2}$ of the form given by Eq. (9). [We have used the symmetry constraint of Eq. (4) as we did earlier when treating scalars. ]

Case (iii), $m<0$ : Here, examination of Eq. (14) shows that we must require as $r \rightarrow 0$

$$
A_{r m}+i A_{\theta m} \sim r^{q}, \text { where } q \geqslant|m|-1
$$

and

$$
A_{r m}-i A_{\theta m} \sim r^{n}, \text { where } p \geqslant|m|+1,
$$

since both these terms occur for $m<0$. Using the same type of argument as for the $m>0$ case above we find here that we must have

$$
\begin{aligned}
& A_{r m}=\lambda_{m m} r^{|m|-1}+r^{|m|+1} g_{m}\left(r^{2}\right), \\
& A_{\theta m}=-i \lambda_{m} r^{|m|-1}+r^{|m|+1} h_{m}\left(r^{2}\right) .
\end{aligned}
$$

Since $A_{z}$ behaves like a scalar, it is described by Eq. ( 8 ). Combining the results derived above we find that the general form for a vector is, for $m \neq 0$,

$$
\begin{aligned}
& A_{r m}=\lambda_{m} r^{|m|-1}+r^{|m|+1} g_{m}\left(r^{2}\right), \\
& A_{\theta m}=i \operatorname{sgn}(m) \lambda_{m} r^{|m|-1} u_{m n}\left(r^{2}\right)+r^{|m|+1} h_{m}\left(r^{2}\right), \\
& A_{z m}=r^{|m|} f_{m}\left(r^{2}\right),
\end{aligned}
$$

while, for $m=0$,

$$
A_{r 0}=r g_{0}\left(r^{2}\right), \quad A_{\theta 0}=r h_{0}\left(r^{2}\right), \quad A_{z 0}=f_{0}\left(r^{2}\right) .
$$

The peculiar form of the $\lambda_{m}$ terms in Eq. (19a) can also be derived by requiring that the divergence and curl of the vector are always finite and then examining these quantities as $r \rightarrow 0$. For example, if we require

$$
\boldsymbol{\nabla} \cdot \mathbf{A}=\text { finite, }
$$

then, as $r \rightarrow 0$, Eq. (20) becomes

$$
\lim _{r \rightarrow 0}\left[\frac{\partial\left(r A_{r}\right)}{\partial r}+\frac{\partial A_{\theta}}{\partial \theta}\right]=0
$$

If we assume that $A_{r} \sim \lambda r^{|m|-1}$, where $m \geqslant 1$, and that both $A_{r}$ and $A_{\theta} \sim \exp (\operatorname{im} \theta)$, then we obtain $A_{\theta}=i \operatorname{sgn}(m) A_{r}$ consistent with Eq. (19a). A similar observation occurs when we calculate $(\boldsymbol{\nabla} \times \mathbf{A})_{2}$. 


\section{IMPORTANCE OF $\boldsymbol{m}=1$ MODES}

Equation (19a) has the very interesting physical consequence of showing that $|m|=1$ Fourier terms form a special class, because only these modes can have $A_{r}$ and $A_{\theta}$ finite at $r=0$. These $|m|=1$ modes are observed to be important in magnetohydrodynamic (MHD) instabilities present in tokamaks, ${ }^{2}$ reversed field pinches, ${ }^{1}$ and spheromaks. ${ }^{3}$

In many physically interesting problems, one is interested in quantities of the form

$$
\hat{z} \cdot \mathrm{A} \times \mathrm{B}=A_{r} B_{\theta}-A_{\theta} B_{r} .
$$

For example, in magnetohydrodynamics, Ohm's law with the Hall term included has the form

$$
\mathbf{E}+\mathbf{U} \times \mathbf{B}-(1 / n e) \mathbf{J} \times \mathbf{B}=\eta \mathbf{J},
$$

where $\mathbf{J}, \mathbf{B}, \mathbf{E}$, and $\mathbf{U}$ are, respectively, the electric current density, the magnetic field, the electric field, and the velocity field; $\eta$ is the resistivity, $n$ is the charged particle density, and $e$ is the charge on an electron. In dynamo theory ${ }^{1,4,5}$ and MHD rf current drive schemes, ${ }^{6,7}$ one searches for ways of driving a dc current on the rhs of Eq. (22) using only ac quantities on the lhs. If this dc current is to be directed in the $z$ direction of a cylindrical coordinate system, then we take the $z$ component of Eq. (22)

$$
\left\langle\widetilde{E}_{z}+\hat{z} \cdot \widetilde{\mathbf{U}} \times \widetilde{\mathbf{B}}-(1 / n e) \hat{z} \cdot \widetilde{\mathbf{J}} \times \widetilde{\mathbf{B}}\right\rangle=\eta J_{z \mathrm{dc}},
$$

where \langle\rangle denotes time average and tilde denotes ac quantity. Since only the $|m|=1$ terms in the $\theta$ and $z$ components of the ac vectors are finite at $r=0$, we immediately know that only $|m|=1$ terms of the ac fields offer the possibility of driving de currents along the $z$ axis. This removes from consideration the infinity of modes for which $|m| \neq 1$ and so provides an enormous simplification ${ }^{1}$ of the problem, even before having to determine the relevant equations for $\mathbf{U}, \mathbf{J}$, B.

\section{ACKNOWLEDGMENTS}

The authors are indebted to J. L. Schwarzmeier, R. A. Nebel, and M. A. Schalit for many stimulating conversations.

This work was supported by the U.S. Department of Energy.

\section{APPENDIX: INFERENCE OF INFINITE DIFFERENTIABILITY FROM A MORE PRIMITIVE ASSUMPTION}

The analysis in the main body of this paper was based on the postulate that all physical quantities when expressed in Cartesian coordinates are infinitely differentiable on the axis. We show here that this postulate may be inferred from a more primitive assumption, namely, the assumption that the location of the cylindrical axis of a coordinate system describing a physical system is arbitrary.

The basic requirement is that the expression for a quantity in terms of a particular set of variables do not exhibit peculiarities which are nonphysical and only a result of the choice of variables. We consider a quantity represented by a nonsingular Fourier series with respect to the angle variable of a cylindrical coordinate system. Suppose on physical grounds that the quantity in question could be represented equally well by a Fourier series with respect to the angle variable of any other cylindrical coordinate system, whose cylindrical axis is parallel to the original axis, but is located anywhere in a neighborhood of the original axis. That is, we suppose that the location of the cylindrical coordinate axis is irrelevant to the physical problem; the location is chosen purely for convenience.

For studying the differentiability at $r=0$ of Fourier series, it suffices to take the case where $f(r, \theta)$ and the coefficients $a_{m}(r)$ are scalars. This is because those quantities can represent an arbitrary Cartesian component of a tensor. Any Fourier series in the variable $\theta$ is manifestly infinitely differentiable with respect to $\theta$ because $\exp (\operatorname{im} \theta$ ) is infinitely differentiable. We now suppose that the quantity represented by $f(r, \theta)$ is nonsingular and could be equally well represented by a Fourier series with respect to the angle variable of any other cylindrical coordinate system whose $z$ axis is parallel to the original $z$ axis, but is located anywhere in a neighborhood of the original axis. This is a weak assumption. If the quantity of physical interest were singular, then either a related nonsingular function could be defined (for example, by subtracting the electric potential due to a line charge located on the cylindrical axis), or the singular quantity could be viewed as the limit of a sequence of nonsingular quantities. The results presented here would apply to each member of the sequence and, therefore, to the singular limit as well.

Consider two equivalent cylindrical coordinate systems, as illustrated in Fig. 1. The coordinates of the chosen cylindrical system are denoted by $(r, \theta, z)$ and the associated Cartesian coordinates are $(x, y, z)$. The $z$ axis is normal to the plane of the drawing and passes through the point labeled $\mathbf{A}$. The coordinates of the other cylindrical system are $\left(r_{n}, \theta_{n}, z\right)$ and the associated Cartesian coordinates are $\left(x_{n}, y_{n}, z\right)$. The axis of that system has coordinates

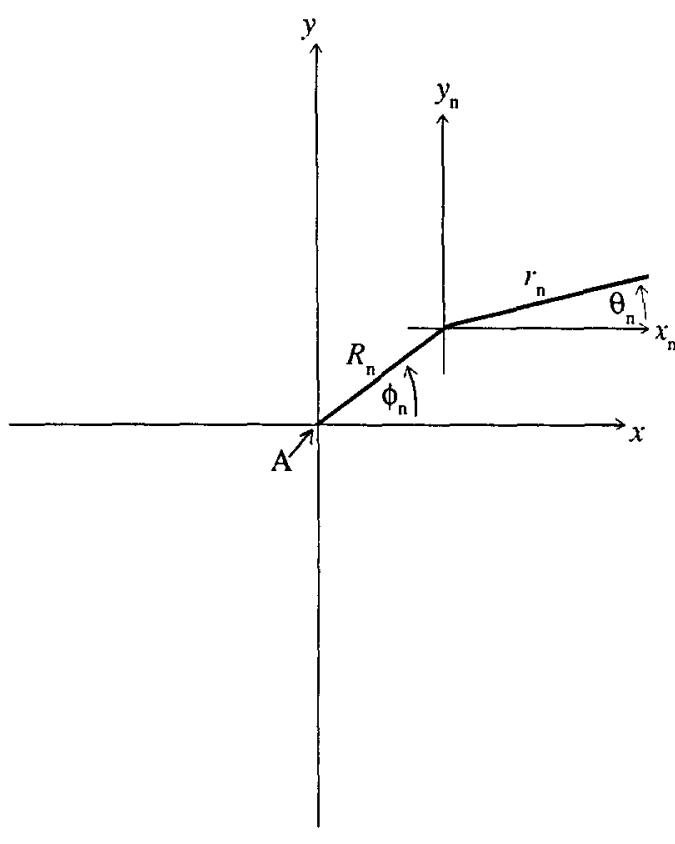

FIG. 1. Relation of the $\left(x_{n}, y_{n}\right)$ coordinate system to the $(x, y)$ coordinate system. 


$$
r=R_{n}, \quad \theta=\phi_{n}
$$

in the chosen cylindrical system; these coordinates are arbitrary. The coordinates $\left(r_{n}, \theta_{n}\right)$ of the axis $A$ are

$$
r_{n}=R_{n}, \quad \theta_{n}=\phi_{n}+\pi \text {. }
$$

Denote the function $f(r, \theta)$ expressed in terms of $\left(r_{n}, \theta_{n}\right)$ by $f_{n}\left(r_{n}, \theta_{n}\right)$ and expressed in terms of $(x, y)$ by $F(x, y)$; by assumption, $f_{n}\left(r_{n}, \theta_{n}\right)$ possesses a Fourier series in the variable $\theta_{n}$ and is thus infinitely differentiable with respect to $\theta_{n}$. The derivatives with respect to $r_{n}$ and $\theta_{n}$ can be expressed in terms of derivatives with respect to $x_{n}$ and $y_{n}$ by repeated application of the formulas

$$
\begin{aligned}
& \frac{\partial}{\partial r_{n}}=\cos \theta_{n} \frac{\partial}{\partial x_{n}}+\sin \theta_{n} \frac{\partial}{\partial y_{n}}, \\
& \frac{\partial}{\partial \theta_{n}}=-r_{n} \sin \theta_{n} \frac{\partial}{\partial x_{n}}+r_{n} \cos \theta_{n} \frac{\partial}{\partial y_{n}} .
\end{aligned}
$$

Since the $\left(x_{n}, y_{n}\right)$ system is related to the $(x, y)$ system by a simple translation, we have

$$
\frac{\partial}{\partial x_{n}}=\frac{\partial}{\partial x}, \frac{\partial}{\partial y_{n}}=\frac{\partial}{\partial y}
$$

and, in particular, we can write Eq. (A4) as

$$
\frac{\partial}{\partial \theta_{n}}=-r_{n} \sin \theta_{n} \frac{\partial}{\partial x}+r_{n} \cos \theta_{n} \frac{\partial}{\partial y} \text {. }
$$

Using Eq. (A6), the derivative

$$
\frac{\partial^{p}}{\partial \theta_{n}^{p}} f_{n}\left(r_{n}, \theta_{n}\right)
$$

can be expressed in terms of derivatives with respect to $x$ and $y$ of $F(x, y)$; that expression will contain the $(p+1)$ derivatives of $F(x, y)$ of order $p$. By choosing $(p+1)$ different axes $\left(R_{n}, \phi_{n}\right)$ and evaluating the $p$ th derivative with respect to $\theta_{n}$ for each of the $(p+1)$ functions $f_{n}\left(r_{n}, \theta_{n}\right)$ at the axis $A$, we obtain a linear system of $(p+1)$ equations for the $(p+1)$ derivatives of $F(x, y)$ of order $p$ at the axis $A$. Solution of that system gives the derivatives of $F(x, y)$ of order $p$ in terms of the derivatives of $F(x, y)$ of lower order and the derivatives

$$
\frac{\partial^{p}}{\partial \theta_{n}^{p}} f_{n}\left(r_{n}, \theta_{n}\right)
$$

all evaluated at the axis $A$. In this way, beginning with $p=1$, we can evaluate all of the derivatives of $F(x, y)$ of order $p$ for each successive value of $p$. The linear system is soluble for nearly any choice of $(p+1)$ axes. For example, for $p=1$, the linear system is

$$
\begin{aligned}
& -R_{1} \sin \left(\phi_{1}+\pi\right) F_{x}(0,0)+R_{1} \cos \left(\phi_{1}+\pi\right) F_{y}(0,0) \\
& =\left.\frac{\partial}{\partial \theta_{1}} f_{1}\left(r_{1}, \theta_{1}\right)\right|_{\substack{1_{1}=R_{1} \\
\theta_{3}=\phi_{1}+\pi}}, \\
& -R_{2} \sin \left(\phi_{2}+\pi\right) F_{x}(0,0)+R_{2} \cos \left(\phi_{2}+\pi\right) F_{y}(0,0) \\
& =\left.\frac{\partial}{\partial \theta_{2}} f_{2}\left(r_{2}, \theta_{2}\right)\right|_{\substack{r_{2}=R_{2} \\
\theta_{2}=\phi_{2}+\pi}} .
\end{aligned}
$$

The determinant of the coefficients is

$$
R_{1} R_{2} \sin \left(\phi_{2}-\phi_{1}\right) \text {, }
$$

so that $F_{x}(0,0)$ and $F_{y}(0,0)$ can be determined from Eqs. (A7) and (A8) as long as $R_{1} \neq 0, R_{2} \neq 0$, and $\phi_{1} \neq \phi_{2}$. By showing how to evaluate all of the derivatives of $F(x, y)$ at the axis $A$, we have shown that $F(x, y)$ is infinitely differentiable at the axis $A$ solely as a result of the arbitrariness of the location of the cylindrical axis of the coordinate system and the postulate of a Fourier representation with respect to the angle variable in each of the possible cylindrical coordinate systems.

'E. J. Caramana, R. A. Nebel, and D. D. Schnack, Phys. Fluids 26, 1305 (1983).

${ }^{2}$ M. N. Rosenbluth, D. A. Monticello, H. R. Strauss, and R. B. White, Phys. Fluids 19, 1987 (1976).

${ }^{3}$ A. Janos, G. W. Hart, and M. Yamada, Phys. Rev. Lett. 55, 2868 (1985).

${ }^{4}$ E. Hotta, M. Suzuki, H. Ohta, and I. Hayashi, Jpn. J. Appl. Phys. 24, 110 (1985).

'M. J. Dutch, A. L. McCarthy, and R. G. Storer, Phys. Rev. Lett. 56, 1563 (1986); M. J. Dutch and A. L. McCarthy, Plasma Phys. Controlled Fusion 28, 695 (1986).

"T. Ohkawa, Comments Plasma Phys. Controlled Fusion 12, 165 (1989).

${ }^{7}$ M. A. Schalit and P. M. Bellan, Phys. Fluids B 1, 2533 (1989). 